\title{
Piecewise Constant Controlled Linear Fuzzy Differential Inclusions
}

\author{
Andrej V. Plotnikov ${ }^{1,2, *}$, Tatyana A. Komleva ${ }^{3}$ \\ ${ }^{1}$ Department of Optimal Control and Economic Cybernetics,Odessa National University named after I.I. Mechnikov, \\ Odessa, 65029, Ukraine \\ ${ }^{2}$ Department of Applied and Calculus Mathematics and CAD,Odessa State Academy of Civil Engineering and Architecture, \\ Odessa, 65026, Ukraine \\ ${ }^{3}$ Department of Mathematics, Odessa State Academy of Civil Engineering and Architecture, \\ Odessa, 65026, Ukraine \\ *Corresponding Author: a-plotnikov@ukr.net
}

Copyright @2013 Horizon Research Publishing All rights reserved.

\begin{abstract}
In this article we consider the some properties of the fuzzy R-solution of the controlled linear fuzzy differential inclusions. Also, many engineering systems use piecewise constant controls. However the majority of results of the theory of optimum control are received for measurable controls. In the given paper we introduce the algorithm of replacement of measurable control on piecewise constant control so that the corresponding fuzzy R-solutions of linear fuzzy systems would be close (with necessary accuracy)
\end{abstract}

Keywords Control, Fuzzy, Linear Differential Inclusion, Optimal Time Problems

\section{Introduction}

In 80th years of XX century the control theory in the conditions of uncertainty was generated. In the given theory, controlled differential inclusions $[9,14,15,22,23$, $24,26]$, the usual controlled differential equations with a set-valued initial condition $[16,38,39]$ or the set-valued differential equations containing control [3, 20, 35] are used at exposition of mathematical model. In recent years, the fuzzy set theory introduced by Zadeh [37] has emerged as an interesting and fascinating branch of pure and applied sciences. The applications of fuzzy set theory can be found in many branches of regional, physical, mathematical, differential equations, and engineering sciences. Recently there have been new advances in the theory of fuzzy differential equations [8, 10, 11, 33] and inclusions $[1,4,5,7,34]$ as well as in the theory of controlled fuzzy differential equations $[6,19,21,28,27,30]$ and inclusions [12, 13, 29, 28, 32].

In this article we consider the some properties of the fuzzy R-solution of the controlled linear fuzzy differential inclusions. Also, in many engineering control systems piecewise constant controls, instead of measurable controls are used. In this paper we prove that for any measurable admissible control $w(\cdot)$ and for any $\varepsilon>0$ there exists piecewise constant admissible control $\bar{w}(\cdot)$ such that the fuzzy R-solutions of the control fuzzy linear system are $\varepsilon$-close.

\section{Preliminaries}

We denote the set of real numbers by $R$ and usual $n$-dimensional space of vectors $x=\left(x^{1}, \ldots, x^{n}\right)$, where $x^{i} \in R, i=\overline{1, n}$, by $R^{n}$. The inner product of two vectors $x$ and $y$ in $R^{n}$ is expressed by $\langle x, y\rangle=$ $x^{1} y^{1}+\cdots+x^{n} y^{n}$. The norm of a vector $x \in R^{n}$ is defined by $\|x\|=<x, x>^{1 / 2}$. Let $\mathcal{C}\left(R^{n}\right)\left(\mathcal{C C}\left(R^{n}\right)\right)$ be the family of all nonempty compact (convex) subsets of $R^{n}$ with the Hausdorff metric

$$
h(A, B)=\max \left\{\max _{a \in A} \min _{b \in B}\|a-b\|, \max _{b \in B} \min _{a \in A}\|a-b\|\right\} .
$$

Let $A \subset R^{n}$ be a compact set. The function $C(A, \psi)=\sup \langle a, \psi\rangle$ is called the support function of $A$, where $\psi \in R^{n}$.

Let $E^{n}$ be the family of mappings $\xi: R^{n} \rightarrow[0,1]$ satisfying the following conditions:

1) $\xi$ is normal, i.e. there exists an $x_{0} \in R^{n}$ such that $\xi\left(x_{0}\right)=1$;

2) $\xi$ is fuzzy convex, i.e. $\xi(\lambda x+(1-\lambda) y) \geq$ $\min \{\xi(x), \xi(y)\}$ whenever $x, y \in \mathrm{R}^{n}$ and $\lambda \in[0,1]$;

3) $\xi$ is upper semicontinuous, i.e. for any $x_{0} \in \mathrm{R}^{n}$ and $\varepsilon>0$ exists $\delta\left(x_{0}, \varepsilon\right)>0$ such that $\xi(x)<\xi\left(x_{0}\right)+\varepsilon$ whenever $\left\|x-x_{0}\right\|<\delta, x \in \mathrm{R}^{n}$;

4) the closure of the set $\left\{x \in \mathrm{R}^{n}: \xi(x)>0\right\}$ is compact.

Let $\widehat{0}$ be the fuzzy mapping defined by $\widehat{0}(x)=0$ if $x \neq 0$ and $\widehat{0}(0)=1$, where $0 \in R^{n}$ is zero vector.

Definition 1. The set $\left\{y \in R^{n}: \xi(y) \geq \alpha\right\}$ is called the $\alpha$ - level $[\xi]^{\alpha}$ of a mapping $\xi \in E^{n}$ for $0<\alpha \leq 1$. The closure of the set $\left\{y \in \mathrm{R}^{n}: \xi(y)>0\right\}$ is called the 0 - level $[\xi]^{0}$ of a mapping $\xi \in \mathrm{E}^{\mathrm{n}}$. 
Let $\xi, \chi \in E^{n}$.

Definition 2. If $[\xi]^{\alpha} \bigcap[\chi]^{\alpha} \neq \emptyset$ for all $\alpha \in[0,1]$, then we say that $\xi \bigcap \chi \neq \emptyset$.

Definition 3. If $[\xi]^{\alpha} \subset[\chi]^{\alpha}$ for all $\alpha \in[0,1]$, then we say that $\xi \subset \chi$.

Theorem 1.[17] If $\xi \in E^{n}$ then

1) $[\xi]^{\alpha} \in \operatorname{conv}\left(R^{n}\right)$ for all $0 \leq \alpha \leq 1$;

2) $[\xi]^{\alpha_{2}} \subset[\xi]^{\alpha_{1}}$ for all $0 \leq \alpha_{1} \leq \alpha_{2} \leq 1$;

3) if $\left\{\alpha_{k}\right\} \subset[0,1]$ is a nondecreasing sequence converging to $\alpha>0$, then $[\xi]^{\alpha}=\bigcap_{k \geq 1}[\xi]^{\alpha_{k}}$.

Conversely, if $\left\{A^{\alpha}: 0 \leq \alpha \leq 1\right\}$ is the family of subsets of $R^{n}$ satisfying the conditions 1) - 3) then there exists $\xi \in \mathrm{E}^{n}$ such that $[\xi]^{\alpha}=A^{\alpha}$ for $0<\alpha \leq 1$ and $[\xi]^{0}=\bigcup_{0<\alpha \leq 1} A^{\alpha} \subset A^{0}$.

Define the metric $D: E^{n} \times E^{n} \rightarrow R_{+}$by the equation $D(\xi, \varsigma)=\sup _{\alpha \in[0,1]} h\left([\xi]^{\alpha},[\varsigma]^{\alpha}\right)$.

Using the results of [36], we know that

1) $\left(E^{n}, D\right)$ is a complete metric space,

2) $D(\xi+\chi, \varsigma+\chi)=D(\xi, \varsigma)$ for all $\xi, \varsigma, \chi \in E^{n}$,

3) $D(k \xi, k \varsigma)=|k| D(\xi, \varsigma)$ for all $\xi, \varsigma \in E^{n}, k \in R$.

Let $I$ be an interval in $R$.

Definition 4. [17] A mapping $f: I \rightarrow E^{n}$ is called continuous at point $t_{0} \in I$ provided for any $\varepsilon>0$ there exists $\delta>0$ such that $D\left(f(t), f\left(t_{0}\right)\right)<\varepsilon$ whenever $\left|t-t_{0}\right|<\delta, t \in I$. A mapping $f: I \rightarrow E^{n}$ is called continuous on $I$ if it is continuous at every point $t_{0} \in I$.

Definition 5. [17] A mapping $f: I \rightarrow E^{n}$ is called measurable on $I$ if for any $\alpha \in[0,1]$ the set-valued mapping $f_{\alpha}(t)=[f(t)]^{\alpha}$ is Lebesgue measurable.

Definition 6. [17] A mapping $f: I \rightarrow E^{n}$ is called integrably bounded on $I$ if there exists a Lebesgue integrable function $k(t)$ such that $\|x\| \leq k(t)$ for all $x \in f_{0}(t), t \in I$.

Definition 7. [17] An element $g \in E^{n}$ is called an integral of $f: I \rightarrow E^{n}$ over $I$ if $[g]^{\alpha}=(A) \int_{I} f_{\alpha}(t) d t$ for any $\alpha \in(0,1]$, where $(A) \int_{I} f_{\alpha}(t) d t$ is the Aumann integral [2].

Theorem 2.[17] If a mapping $f: I \rightarrow E^{n}$ is measurable and integrably bounded then $f$ is integrable over I.

Now, we consider the following controlled fuzzy system

$$
\dot{x} \in F(t, x, u), \quad x(0)=x_{0},
$$

where $x \in R^{n} ; t \in R_{+} ; u \in U \subset R^{k}$ is a control parameter; $F: R_{+} \times R^{n} \times R^{k} \rightarrow E^{n}$ is a fuzzy mapping, $x_{0} \in R^{n}$.

By an admissible control we mean any measurable function $u: R_{+} \rightarrow R^{k}$ satisfying the inclusion $u(t) \in U$ everywhere. We denote the set of all admissible control of controlled fuzzy system $(1)$ by $\mathcal{U}\left(R_{+}\right)$(or $\mathcal{U}$ ).

Obviously, the controlled fuzzy differential inclusion (1) turns into the ordinary fuzzy differential inclusion

$$
\dot{x} \in G(t, x), \quad x(0)=x_{0},
$$

if the control $\tilde{u}(\cdot) \in \mathcal{U}$ is fixed and $G(t, x) \equiv F(t, x, \tilde{u}(t))$.

We interpret $[1,4,5]$ the fuzzy differential inclusion (2) as a family of differential inclusions

$$
\dot{x}_{\alpha} \in G_{\alpha}\left(t, x_{\alpha}(t)\right) \equiv\left[G\left(t, x_{\alpha}(t)\right)\right]^{\alpha}, x_{\alpha}(0)=x_{0},
$$

where the subscript $\alpha$ indicates that the $\alpha$-level set of a fuzzy set is involved (the system (3) can only have any significance as a replacement for (1) if the solutions generate fuzzy sets (fuzzy R-solution) [7]).

Let $X(t)$ denotes the fuzzy R-solution of the differential inclusion (2), then $X(t, \tilde{u})$ denotes the fuzzy Rsolution of the controlled differential inclusion (1) for the fixed $\tilde{u}(\cdot) \in \mathcal{U}$.

Definition 8. The set $Y(T)=\{X(T, u) \mid u(\cdot) \in \mathcal{U}\}$ is called the attainable set of the fuzzy system (1).

\section{The controlled fuzzy differen- tial inclusion}

In this article we consider linear controlled differential equations with fuzzy parameter

$$
\dot{x}_{\alpha}=A(t) x_{\alpha}+\phi\left(t, u, v_{\alpha}(t)\right), \quad x_{\alpha}(0)=x_{0},
$$

where $x_{\alpha} \in R^{n} ; t \in R_{+} ; A(t): R_{+} \rightarrow R^{n \times n}$ is a matrix $(n \times n) ; \phi(t, u, v): R_{+} \times R^{k} \times R^{m} \rightarrow R^{n}$ is a function; $u \in U \subset R^{k}$ is a control parameter; $v_{\alpha}(\cdot)$ is a measurable function such that $v_{\alpha}(t) \in[V]^{\alpha}, V \in E^{m}$ for every $t \geq 0$; $x_{0} \in R^{n}$.

In correspondence to the controlled problem (4) we will put the following controlled differential inclusion with fuzzy right-hand side

$$
\dot{x} \in A(t) x+f(t, u), \quad x(0)=x_{0},
$$

where $f: R_{+} \times R^{k} \rightarrow E^{n}$ is a fuzzy mapping, such that $[f(t, u)]^{\alpha} \equiv \varphi\left(t, u,[V]^{\alpha}\right)$ for all $\alpha \in[0,1]$.

Theorem 4. [27] Let the following conditions hold:

i) all elements of the matrix $A(t)$ are measurable on the interval $[0, T]$;

ii) there exists $a>0$ such that $\|A(t)\| \leq$ a for almost every $t \in[0, T]$

iii) the set $U$ is a nonempty compact convex subset of the space $R^{k}$, i.e. $U \in \mathcal{C C}\left(R^{k}\right)$;

iv) the fuzzy mapping $f(t, u)$ is measurable on the interval $[0, T]$ and continuous on the set $U$;

$v$ ) there exists $b>0$ such that $\|f(t, u)\| \leq b$ for almost every $t \in[0, T]$ and for every $u \in U$;

vi) the set $Q(t)=\{f(t, u(t)): u(\cdot) \in \mathcal{U}\}$ is compact and convex for almost every $t \in[0, T]$.

Then

1) the fuzzy $R$-solution $X(t, u)$ of the inclusion (5) such that

$$
X(t, u)=\Phi(t) x_{0}+\Phi(t) \int_{0}^{t} \Phi^{-1}(s) f(s, u(s)) d s
$$

for all $t \in[0, T]$ and $u(\cdot) \in \mathcal{U}$;

2) $X(t, u) \in E^{n}$ for all $t \in[0, T]$ and $u(\cdot) \in \mathcal{U}$;

3) the fuzzy $R$-solution $X(\cdot, u)$ is continuous on the interval $[0, T]$ for all $u(\cdot) \in \mathcal{U}$;

4) the attainable set $Y(T)$ is nonempty convex compact subset of the space $E^{n}$, i.e. $Y(T) \in \mathcal{C C}\left(E^{n}\right)$, where $\Phi(\cdot)$ is a fundamental matrix of the differential equation $\dot{x}=A(t) x$ such that $\Phi(0)=I, I$ is a unit matrix.

Remark 1. Properties of space $\mathcal{C}\left(E^{n}\right)$ have been considered in work [34]. 
We obtained the basic properties of the fuzzy Rsolution of systems (5).

In many engineering controlled systems piecewise constant controls, instead of measurable controls are used. Let $U=\prod_{i=1}^{m}\left[u_{\min }^{i}, u_{\max }^{i}\right]$. Now, we need to establish that for any measurable admissible control $u(\cdot)$ and for any $\varepsilon>0$ there exists piecewise constant admissible control $\bar{u}(\cdot)$ such that for fuzzy R-solutions of system (5) holds $D(X(t, u), X(t, \bar{u}))<\varepsilon$ for all $t \in[0, T]$.

Theorem 5. Let the conditions of the theorem 4 fulfill, and

7) There exists constant $\gamma>0$ such that

$$
\begin{gathered}
D\left(\int_{0}^{t} f\left(s, u_{1}(s)\right) d s, \int_{0}^{t} f\left(s, u_{2}(s)\right) d s\right) \leq \\
\leq \gamma\left\|\int_{0}^{t} u_{1}(s) d s-\int_{0}^{t} u_{2}(s) d s\right\|
\end{gathered}
$$

for all $u_{1}(\cdot), u_{2}(\cdot) \in \mathcal{U}$ and $t \in[0, T]$.

Then for every $u(\cdot) \in \mathcal{U}$ there exists $\bar{u}(\cdot) \in \mathcal{U}$ such that

1) $\bar{u}(t)$ is constant on every $\left[(i-1) \frac{T}{k}, i \frac{T}{k}\right), i=\overline{1, k}$;

2) $\bar{u}_{i}(t)=\left\{\left(\bar{u}_{i}^{1}(t), \ldots, \bar{u}_{i}^{m}(t)\right)^{T} \mid \bar{u}_{i}^{j}(t) \quad \in\right.$ $\left.\left\{u_{\text {min }}^{j}, u_{\text {max }}^{j}\right\}, i=\overline{1, k}, j=\overline{1, m}\right\}$ for every $t \in[0, T]$;

3) $D(X(t, u), X(t, \bar{u})) \leq \gamma e^{a T} \frac{T}{2 k}\left\|u_{\max }-u_{\min }\right\|$ for all $t \in[0, T]$, where $u_{\min }=\left(u_{\min }^{1}, \ldots, u_{\min }^{m}\right), u_{\max }=$ $\left(u_{\max }^{1}, \ldots, u_{\max }^{m}\right)$.

Proof. Choose any $u(\cdot) \in \mathcal{U}$ and any $k \in N$. Let $W_{i}=\left(W_{i}^{1}, \ldots, W_{i}^{m}\right)^{T}$, where $W_{i}^{j}=\int_{0}^{i \frac{T}{k}} u^{j}(s) d s, i=\overline{1, k}$, $j=\overline{1, m}$. Obviously, $W_{i+1}^{j}-W_{i}^{j}=\int_{\frac{i T}{k}}^{\frac{(i+1) T}{k}} u^{j}(s) d s$, $u_{\min }^{j} \frac{T}{k} \leq W_{i+1}^{j}-W_{i}^{j} \leq u_{\max }^{j} \frac{T}{k}, j=\overline{1, m}$, and $\left\|W_{i+1}-W_{i}\right\| \leq\left\|u_{\max }-u_{\min }\right\| \frac{T}{k}$.

Now we take

$$
\bar{u}(t)= \begin{cases}\bar{u}_{1}, & t \in\left[0, \frac{T}{k}\right), \\ \vdots & \vdots \\ \bar{u}_{k-1}, & t \in\left[\frac{(k-2) T}{k}, \frac{(k-1) T}{k}\right), \\ \bar{u}_{k}, & t \in\left[\frac{(k-1) T}{k}, T\right],\end{cases}
$$

such that

1) $\bar{u}_{1}=\left(\bar{u}_{1}^{1}, \ldots, \bar{u}_{1}^{m}\right)^{T}$, where

$\bar{u}_{1}^{j}= \begin{cases}u_{\text {max }}^{j}, & \text { if } W_{1}^{j} \geq \frac{T}{2 k}\left(u_{\text {max }}^{j}+u_{\text {min }}^{j}\right), \quad j=\overline{1, m} ; \\ u_{\text {min }}^{j}, & \text { if } W_{1}^{j}<\frac{T}{2 k}\left(u_{\text {max }}^{j}+u_{\text {min }}^{j}\right),\end{cases}$

2) $\bar{u}_{i}=\left(\bar{u}_{i}^{1}, \ldots, \bar{u}_{i}^{m}\right)^{T}, i=\overline{2, k}$, where

$\bar{u}_{i}^{j}= \begin{cases}u_{\text {max }}^{j}, & \text { if } W_{i}^{j}-\sum_{l=1}^{i-1} \bar{u}_{j}^{l} \frac{T}{k} \geq \frac{T}{2 k}\left(u_{\text {max }}^{j}+u_{\text {min }}^{j}\right), \\ u_{\text {min }}^{j}, & \text { if } W_{i}^{j}-\sum_{l=1}^{i-1} \bar{u}_{j}^{l} \frac{T}{k}<\frac{T}{2 k}\left(u_{\text {max }}^{j}+u_{\text {min }}^{j}\right),\end{cases}$

$j=\overline{1, m}$

Obviously, for $i=1$ and $j=\overline{1, m}$ we have

a) if $\bar{u}_{1}^{j}=u_{\text {max }}^{j}$, when

$$
-\frac{T}{2 k}\left(u_{\max }^{j}-u_{\min }^{j}\right) \leq W_{1}^{j}-\bar{u}_{1}^{j} \frac{T}{k} \leq 0
$$

b) if $\bar{u}_{1}^{j}=u_{\min }^{j}$, when

$$
\frac{T}{2 k}\left(u_{\max }^{j}-u_{\min }^{j}\right)>W_{1}^{j}-\bar{u}_{1}^{j} \frac{T}{k} \geq 0 .
$$

Hence we obtain $\left|W_{1}^{j}-\bar{u}_{1}^{j}\right| \leq \frac{T}{2 k}\left(u_{\max }^{j}-u_{\min }^{j}\right), j=$ $\overline{1, m}$, and $\left\|W_{1}-\bar{u}_{1}\right\| \leq \frac{T}{2 k}\left\|u_{\max }-u_{\min }\right\|$.

Thus, by induction, we obtain that, for $i=\overline{2, k}$

$$
\left|W_{i}^{j}-\sum_{l=1}^{i} \bar{u}_{l}^{j} \frac{T}{k}\right| \leq \frac{T}{2 k}\left(u_{\max }^{j}-u_{\min }^{j}\right), j=\overline{1, m},
$$

and

$$
\left\|W_{i}-\sum_{l=1}^{i} \bar{u}_{l} \frac{T}{k}\right\| \leq \frac{T}{2 k}\left\|u_{\max }-u_{\min }\right\| .
$$

Therefore, if $t_{i}=\frac{i T}{k}, i=\overline{1, k}$; then

$$
\left\|\int_{0}^{t_{i}} u(s) d s-\int_{0}^{t_{i}} \bar{u}(s) d s\right\| \leq \frac{T}{2 k}\left\|u_{\max }-u_{\min }\right\| .
$$

Now, we take $t \in\left(\frac{(i-1) T}{k}, \frac{i T}{k}\right)$. Then

$$
\begin{gathered}
\left\|\int_{0}^{t} u(s) d s-\int_{0}^{t} \bar{u}(s) d s\right\| \leq \\
\leq\left\|W_{i-1}-\sum_{l=1}^{i-1} \bar{u}_{l} \frac{T}{k}+\int_{\frac{(i-1) T}{k}}^{t}\left(u(s)-\bar{u}_{i}\right) d s\right\| .
\end{gathered}
$$

As for all $j=\overline{1, m}$

$$
\begin{gathered}
W_{i}^{j}-\sum_{l=1}^{i} \bar{u}_{l}^{j} \frac{T}{k} \geq \\
\geq W_{i-1}^{j}-\sum_{l=1}^{i-1} \bar{u}_{l}^{j} \frac{T}{k}+\int_{\frac{(i-1) T}{k}}^{t}\left(u^{j}(s)-\bar{u}_{i}^{j}\right) d s \geq \\
\geq W_{i-1}^{j}-\sum_{l=1}^{i-1} \bar{u}_{l}^{j} \frac{T}{k} ; \\
\text { then }\left\|\int_{0}^{t} u(s) d s-\int_{0}^{t} \bar{u}^{t}(s) d s\right\| \leq{ }^{i} \| \overline{m a x}_{l=1}\left\{W_{i}-\sum_{l=1}^{T}\|,\| W_{i-1}^{j}-\sum_{l=1}^{i-1} \bar{u}_{l}^{j} \frac{T}{k} \|\right\} .
\end{gathered}
$$

By (8), we get for all $t \in[0, T]$

$$
\left\|\int_{0}^{t} u(s) d s-\int_{0}^{t} \bar{u}(s) d s\right\| \leq \frac{T}{2 k}\left\|u_{\max }-u_{\min }\right\| .
$$

Now, applying condition 1) of the theorem 4 and condition 7 ) of the theorem 5 , we obtain

$$
\begin{gathered}
D(X(t, u), X(t, \bar{u}))= \\
=D\left(\int_{0}^{t} \Phi(t) \Phi^{-1}(s) f(s, u(s)) d s\right.
\end{gathered}
$$




$$
\begin{gathered}
\left.\int_{0}^{t} \Phi(t) \Phi^{-1}(s) f(s, \bar{u}(s)) d s\right) \leq \\
\leq \varphi_{1} \varphi_{2} D\left(\int_{0}^{t} f(s, u(s)) d s, \int_{0}^{t} f(s, \bar{u}(s)) d s\right) \leq \\
\leq \varphi_{1} \varphi_{2} \gamma\left\|\int_{0}^{t} u(s) d s-\int_{0}^{t} \bar{u}(s) d s\right\|,
\end{gathered}
$$

where $\varphi_{1}=\sup _{t \in[0, T]}\|\Phi(t)\|, \varphi_{2}=\sup _{t \in[0, T]}\left\|\Phi^{-1}(t)\right\|$, i.e. we obtain

$$
D(X(t, w), X(t, \bar{w})) \leq \varphi_{1} \varphi_{2} \gamma\left\|\int_{0}^{t} u(s) d s-\int_{0}^{t} \bar{u}(s) d s\right\| .
$$

By (9), we have

$$
D(X(t, u), X(t, \bar{u})) \leq \varphi_{1} \varphi_{2} \gamma \frac{T}{2 k}\left\|u_{\max }-u_{\min }\right\|
$$

This concludes the proof.

Remark 2. Obviously, if we take

$$
k>\gamma \varphi_{1} \varphi_{2} \frac{T}{2 \varepsilon}\left\|u_{\max }-u_{\min }\right\|
$$

then $D(X(t, u), X(t, \bar{u}))<\varepsilon \quad$ for all $t \in[0, T]$.

Here we consider the numerical solution of problem by proposed approach in above.

Example. Let

$$
\dot{x} \in x+B w+S, x(0) \in X_{0},
$$

where $x \in R^{2}, B=\left(\begin{array}{ll}1 & 0 \\ 0 & 1\end{array}\right), S \in E^{2}$ such that $[S]^{\alpha}=S_{1-\alpha}(0)$ for all $\alpha \in[0,1], X_{0} \in E^{2}$ such that $\left[X_{0}\right]^{\alpha}=S_{2-2 \alpha}(0)$ for all $\alpha \in[0,1], w=\left(w_{1}, w_{2}\right)^{T} \in U \in$ $\operatorname{conv}\left(R^{2}\right)$ is the control, $U=[-1,1] \times[1,3], T=0.504$, $\varepsilon=0.1$.

Let $w_{1}(t)=\sin (10 t), w_{2}(t)=2-\cos (5 t)$. By remark 2 , we get $k>e^{0.504} \frac{0.504}{2 \cdot 0.1} 2 \sqrt{2} \approx 11.799$, i.e. $k=12$. Then, by construction (6), we obtain

$$
\begin{aligned}
& \bar{w}_{1}(t)= \begin{cases}-1, & t \in I_{i}, i \in\{1,2,8,9,11,12\}, \\
1, & t \in I_{j}, j \in\{3,4,5,6,7,10\},\end{cases} \\
& \bar{w}_{2}(t)= \begin{cases}1, & t \in I_{i}, i \in\{1,2,3,4,5,7,9\}, \\
3, & t \in I_{j}, j \in\{6,8,10,11,12\},\end{cases}
\end{aligned}
$$

where $I_{i}=\left[\frac{0.504(i-1)}{12}, \frac{0.504 i}{12}\right], i=\overline{1,12}$. In this case, $D(x(t, w), x(t, \bar{w}))<0.1$ for all $t \in[0,0.504]$.

\section{Conclusions}

In the last years, a number of works devoted to problems of optimal control of set-valued trajectories (fuzzy trajectories, trajectory bundles or an ensemble of trajectories) appeared. These works fall into a subdivision of the optimal control theory, namely, the theory of process control under uncertainty and fuzzy conditions. This is conditioned by the fact that, in actual problems arising in economy and engineering in the course of construction of a mathematical model, it is practically impossible to exactly describe the behavior of an object. It is explained by following facts. First, for some parameters of the object, it impossible to specify exact values and laws of their change, but it is possible to determine the domain of these changes. Second, for the sake of simplicity of the mathematical model being constructed, the equations that describe the behavior of the object are simplified and one should estimate the consequences of such a simplification. Therefore, if is possible to divide the articles devoted to this direction into two types characterized by the following distinctive features:

1) there exists an incomplete or fuzzy information on the initial data;

2) the equations describing the behavior of the object to be controlled are assumed to be inexact, for example, they can contain some parameters whose exact values and laws of variation are unknown but the domain of their values is fuzzy.

In the second case, fuzzy differential inclusions are frequently used to describe behavior of objects. The reason is that, first this approach is most obvious and, second, theory of fuzzy and ordinary differential inclusions is well found and is rapidly developed at the present time.

In this article, properties of R-solutions of controlled fuzzy linear differential inclusions are received, also we introduce the algorithm of replacement of measurable control on piecewise constant control so that the corresponding fuzzy R-solutions of linear fuzzy systems would be close (with necessary accuracy).

In the first, as to receive an exact solution for the fuzzy differential equations it is impossible, this result helps to construct the approached solutions for these equations.

In the second, the obtained result is useful for constructing the $\varepsilon$-optimal piecewise constant controls for optimal control fuzzy system (fuzzy Mayer problem [25], fuzzy time-optimal problem $[29,30,31,32]$ and other).

\section{REFERENCES}

[1] J.-P. Aubin. Fuzzy differential inclusions. Probl. Control Inf. Theory, 1990, 19(1): $55-67$.

[2] R.J. Aumann. Integrals of set-valued functions. J. Math. Anal. Appl., 1965, 12: 1 - 12, doi:10.1016/0022247X(65)90049-1.

[3] A.V. Arsirii, A.V. Plotnikov. Systems of control over set-valued trajectories with terminal quality criterion. Ukr. Math. J., 2009, 61(8): 1349 - 1356, doi:10.1007/s11253-010-0280-3.

[4] V.A. Baidosov. Differential inclusions with fuzzy righthand side. Soviet Mathematics, 1990, 40(3): 567 - 569.

[5] V.A. Baidosov. Fuzzy differential inclusions. J. of Appl. Math. and Mechan., 1990, 54(1): 8 - 13, doi:10.1016/0021-8928(90)90080-T.

[6] T.E. Dabbous. Adaptive control of nonlinear systems using fuzzy systems. J. Ind. Manag. Optim., 2010, 6(4): 861 - 880, doi:10.3934/jimo.2010.6.861.

[7] E. Hüllermeier. An approach to modelling and simulation of uncertain dynamical system. Int. J. Uncertain. Fuzziness Knowl.-Based Syst., 1997, 7: 117 - 137, doi:10.1142/S0218488597000117. 
[8] O. Kaleva. Fuzzy differential equations. Fuzzy Sets Syst., 1987, 24(3): 301 - 317, doi:10.1016/01650114(87)90029-7.

[9] N. Kikuchi. On contingent equations. Japan-United States Seminar on Ordinary Differential and Functional Equations, Kyoto, 1971, Lecture Notes in Math., Springer, Berlin, 1971, 243: 169 - 181, doi:10.1007/BFb0058726.

[10] V. Lakshmikantham, Gnana T. Bhaskar, Devi J. Vasundhara. Theory of Set Differential Equations in Metric Spaces. Cambridge Scientific Publishers, Cambridge, 2006.

[11] V. Lakshmikantham, R. Mohapatra. Theory of Fuzzy Differential Equations and Inclusions. Taylor - Francis, 2003.

[12] I.V. Molchanyuk, A.V. Plotnikov. Linear control systems with a fuzzy parameter. Nonlinear Oscil. (N. Y.), 2006, 9(1): 59 - 64, doi:10.1007/s11072-006-0025-2.

[13] I.V. Molchanyuk, A.V. Plotnikov. Necessary and sufficient conditions of optimality in the problems of control with fuzzy parameters. Ukr. Math. J., 2009, 61(3): 457 - 463, doi:10.1007/s11253-009-0214-0.

[14] S. Otakulov. A minimax control problem for differential inclusions. Soviet Math. Dokl., 1988, 36(2): 382 - 387.

[15] S. Otakulov. On the approximation of the timeoptimality problem for controlled differential inclusions. Cybernet. Systems Anal., 1994, 30(3): 458 - 462, doi:10.1007/BF02366480.

[16] D.A. Ovsyannikov. Mathematical methods for the control of beams. Leningrad. Univ., 1980.

[17] J.Y. Park, H.K. Han. Existence and uniqueness theorem for a solution of fuzzy differential equations. Int. J. Math. Math. Sci., 1999, 22(2): 271 - 279, doi:10.1155/S0161171299222715.

[18] J.Y. Park, H.K. Han. Fuzzy differential equations. Fuzzy Sets Syst., 2000, 110(1): 69 - 77, doi:10.1016/S0165-0114(98)00150-X.

[19] N.D. Phu, T.T. Tung. Some properties of sheafsolutions of sheaf fuzzy control problems. Electron. J. Differ. Equ., 2006, (108): 8 pp., (electronic) URL: http://ejde.math.txstate.edu.

[20] N.D. Phu, T.T. Tung. Some results on sheaf-solutions of sheaf set control problems. Nonlinear Analysis, 2007, 67(5): 1309 - 1315, doi:10.1016/j.na.2006.07.018.

[21] N.D. Phu, T.T. Tung. Existence of solutions of fuzzy control differential equations. J. Sci. Tech. Devel., 2007, 10(5): $5-12$.

[22] A.V. Plotnikov. A problem of control of bundles of trajectories. Siberian Math. J., 1992, 33(2): 351 - 354, doi:10.1007/BF00971112.

[23] A.V. Plotnikov. Two control problems under uncertainty conditions. Cybernet. Systems Anal., 1993, 29(4): 567 - 573, doi:10.1007/BF01125871.
[24] A.V. Plotnikov. Linear problems of optimal control of multiple-valued trajectories. Cybern. Syst. Anal., 2002, 38(5): 772 - 782, doi:10.1023/A:1021899111846.

[25] A.V. Plotnikov. The averaging of control linear fuzzy differential equations. J. Adv. Res. Appl. Math., 2011, 3(3): 1 - 20, 2011, doi: 10.5373/jaram.664.120610.

[26] A.V. Plotnikov, T.A. Komleva. Some properties of trajectory bunches of controlled bilinear inclusion. Ukr. Math. J., 2004, 56(4): 586 - 600, doi:10.1007/s11253005-0114-x.

[27] A.V. Plotnikov, T.A. Komleva. Linear problems of optimal control of fuzzy maps. Intelligent Information Management, 2009, 1(3): 139 - 144, doi:10.4236/iim.2009.13020.

[28] A.V. Plotnikov, T.A. Komleva. Fuzzy quasidifferential equations in connection with the control problems. Int. J. Open Problems Compt. Math., 2010, 3(4): 439 - 454.

[29] A.V. Plotnikov, T.A. Komleva, A.V. Arsiry. Necessary and sufficient optimality conditions for a control fuzzy linear problem. Int. J. Industrial Mathematics, 2009, 1(3): $197-207$.

[30] A.V. Plotnikov, T.A. Komleva, I.V. Molchanyuk. Linear control problems of the fuzzy maps. J. Software Engineering \& Applications, 2010, 3(3): 191 - 197, doi:10.4236/jsea.2010.33024.

[31] A.V. Plotnikov, T.A. Komleva, I.V. Molchanyuk. The time-optimal problems for controlled fuzzy R-solutions. Intelligent Control and Automation, 2011, (2): 152 159, doi:10.4236/ica.2011.22018.

[32] A.V. Plotnikov, T.A. Komleva, I.V. Molchanyuk. Linear control differential inclusions with fuzzy right-hand side and some optimal problems, Journal Advanced Research in Dynamical and Control Systems, 2011, 3 (2): $34-46$.

[33] A.V. Plotnikov, N.V. Skripnik. Differential Equations with "Clear" and Fuzzy Multivalued Right-Hand Sides. Asymptotics Methods. AstroPrint, Odessa, 2009.

[34] A.V. Plotnikov, N.V. Skripnik. The generalized solutions of the fuzzy differential inclusions. Int. J. Pure Appl. Math., 2009, 56(2): 165 - 172.

[35] V.A. Plotnikov, O.D. Kichmarenko. Averaging of controlled equations with the Hukuhara derivative. Nonlinear Oscil. (N. Y.), 2006, 9(3): 365 - 374, doi:10.1007/s11072-006-0050-1.

[36] M.L. Puri, D.A. Ralescu. Fuzzy random variables. J. Math. Anal. Appl., 1986, 114(2): 409 - 422, doi:10.1016/0022-247X(86)90093-4.

[37] L.A. Zadeh. Fuzzy sets. Inf. Contr., 1965, (8): 338-353, doi:10.1016/S0019-9958(65)90241-X.

[38] V.I. Zubov. Dynamics of controlled systems. Vyssh. Shkola, Moscow, 1982.

[39] V.I. Zubov. Stability of motion. Lyapunov methods and their application. Vyssh. Shkola, Moscow, 1984. 